\title{
Dysphagia Lusoria: A Case Report
}

\author{
Nik Qisti F, Shahrun Niza AS, Razrim R \\ Department of Surgery, Universiti Kebangsaan Malaysia Medical Centre, Kuala Lumpur, Malaysia
}

\begin{abstract}
Aberrant right subclavian artery is a rare cause of dysphagia. This is a congenital anomaly with the right subclavian artery originating from the dorsal part of the aortic arch and coursing through the mediastinum between the esophagus and the vertebral column. We report a case of a patient with chronic dysphagia caused by this condition. We further discuss the case with regards to its clinical features and options of management.
\end{abstract}

Keywords: Dysphagia, barium swallow, aberrant right subclavian artery

\section{INTRODUCTION}

Of all causes of dysphagia, compression of the oesophagus by a blood vessel is very rare. In 1794, David Bayford first discovered the condition, accidentally, after the necropsy of a 62-year old lady who suffered from chronic dysphagia. ${ }^{1} \mathrm{He}$ coined the condition as "dysphagia lusus naturae" translated from Latin as "difficulty in swallowing, freak of nature". The dysphagia was caused by an aberrant right subclavian artery compressing the oesophagus posteriorly.

This anomaly is the most common developmental anomaly of the aortic arch, with an incidence ranging from $0.5 \%$ to $1.8 \% .^{2}$ People with this anomaly are usually asymptomatic; however, in some dysphagia occurs. In this case report, we describe a lady with a chronic history of dysphagia who we eventually came up with the diagnosis of Dysphagia Lusoria. The presentation, anatomical anomaly, investigations and treatment options of this rare entity will be discussed in further detail.

\section{CASE REPORT}

A 66-year-old woman presented to the general surgical clinic with epigastric discomfort and intermittent dysphagia. The symptoms started in her teenage years and have subsided as she

Corresponding author:

Dr Shahrun Niza b. Abdullahi Suhaimi, Consultant General Surgeon,

Department of Surgery,

University Kebangsaan Malaysia Medical Centre, Jalan Yaakob Latif, Bandar Tun Razak, Cheras, 56000, Kuala Lumpur, Malaysia. Email address: shahrun72.sn@gmail.com

Phone No: +6013332 3773 progressed into adulthood. She was first seen in the gastroenterology clinic in 1994 for which she had an oesophagogastroduodenoscopy (OGDS) that showed grade 1 hiatus hernia with antral gastritis. She was treated with proton pump inhibitors and prokinetics. After 2 months following treatment her symptoms improved. Her past medical history includes bronchial asthma with underlying bronchiectasis, hypertension, dyslipidaemia.

After 10 years her symptoms recurred and her dysphagia worsened from solids to fluids. There were no other constitutional symptoms. She was referred to the surgical outpatient clinic as she has failed medical therapy initiated by the gastroenterologists. On examination, she was not cachectic. Her vital signs were normal and she has a BMI of 20.3. She was pink and was not jaundiced.

Per abdominal examination was unremarkable. Her sodium was $141 \mathrm{mmol} / \mathrm{L}$, potassium was $3.8 \mathrm{mmol} / \mathrm{L}$, urea $5.6 \mathrm{mmmol} / \mathrm{L}$ and creatinine was $43 \mathrm{umol} / \mathrm{L}$ all of which were unremarkable. Her albumin was $45 \mathrm{~g} / \mathrm{L}$, bilirubin $5 \mathrm{umol} / \mathrm{L}$, alanine aminotransferase $36 \mathrm{U} / \mathrm{L}$, alkaline phosphatase $102 \mathrm{U} / \mathrm{L}$, calcium 2.43 $\mathrm{mmol} / \mathrm{L}$ and phosphate $1.47 \mathrm{mmol} / \mathrm{L}$. A full neurological assessment was unremarkable. A repeat OGDS was performed and showed mild antral gastritis and a hiatus hernia consistent with the previous findings. There were no intraluminal lesions or strictures.

Following upper endoscopy, we proceeded with a barium swallow study. It showed a persistent indentation at the proximal third of the oesophagus on lateral and anterior views. The abnormality was situated superior to the arch of the aorta at approximately the level of T4 and T5 vertebrae. This is suggestive of an anomalous right subclavian artery (figure 1). 

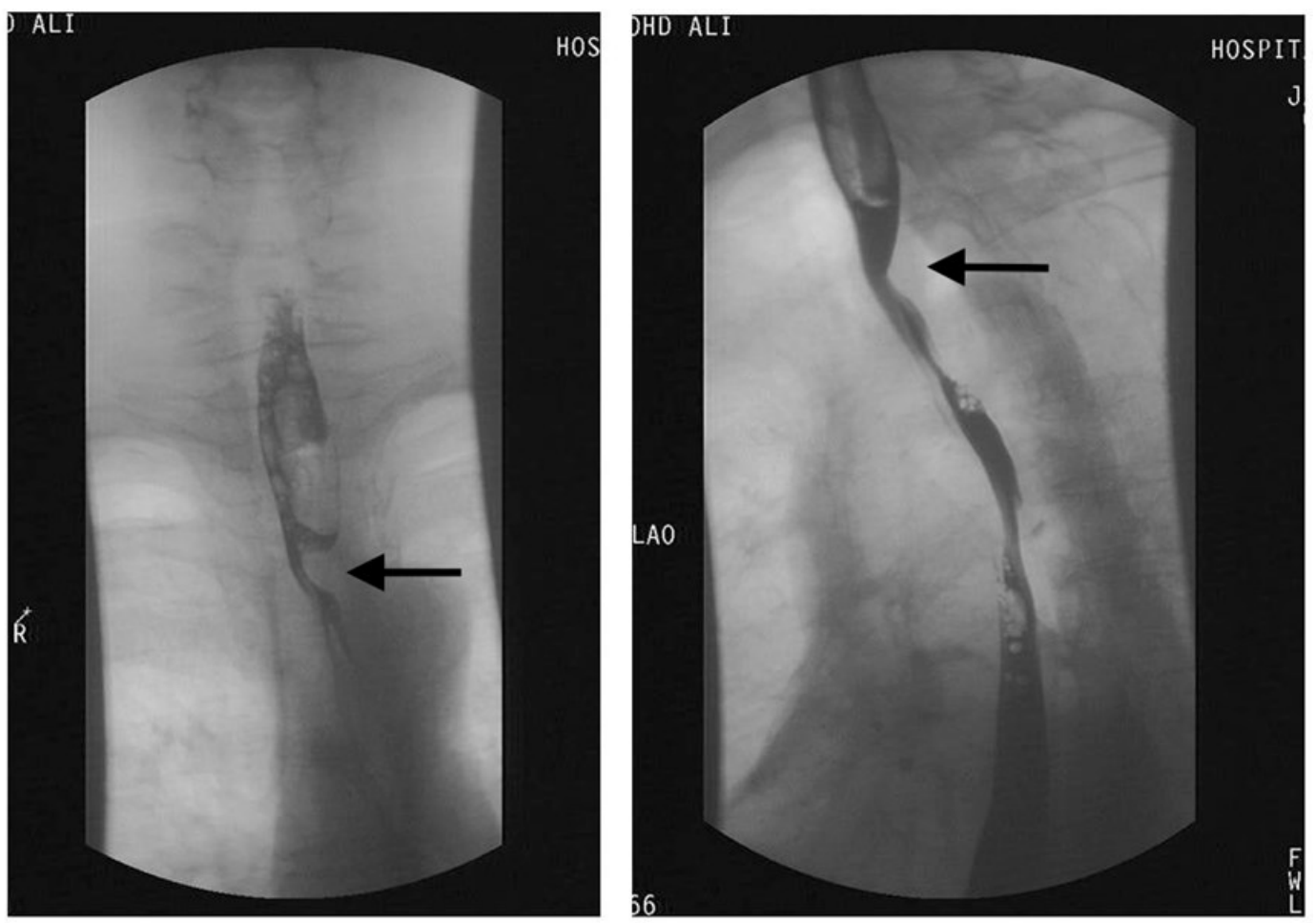

Figure 1: A barium swallow study showing an oblique ascending external compression at the level of T4 vertebrae characteristic of a compression by the lusorian artery. Image on the left is the posterioranterior view with a clear compression represented by arrow. Image on the right is the lateral view with an arrow indicating the same posterior compression.

A Computed Tomography (CT) aortogram was performed to further delineate the aorta and its major branches. The right subclavian artery was seen to arise distal to the left subclavian artery from the arch of aorta. The vessel curved posterior to the oesophagus before continuing its course to the right side of the body (figure 2). The rest of the aortic arch branches were of normal configuration. The features were compatible with an anomalous right subclavian artery or dysphagia lusoria.

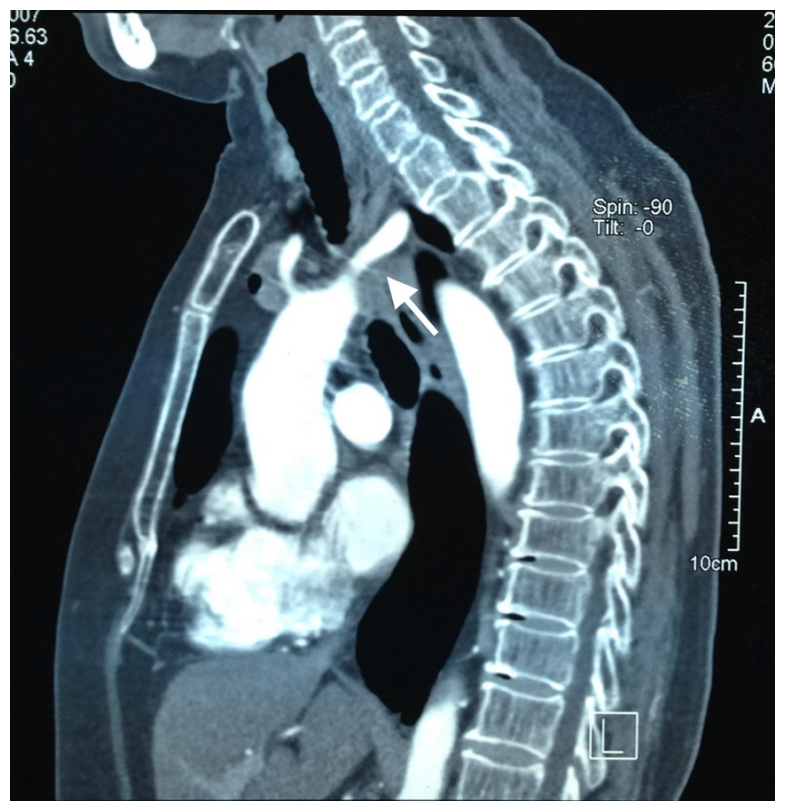

Figure 2: A sagital view of a CT scan with contrast in the arterial phase demonstrating the compression of the oesophagus, posteriorly, as a consequence of the abberatnt right subclavian artery.
In view of the above findings, the patient was advised to undergo some dietary modifications to include more semisolid foods and was started on proton pump inhibitors. She was also prescribed pro-motility agents to help aid relieving her reflux symptoms from the hiatus hernia. She was counselled for the possibility of surgical treatment in the event of intractable symptoms should medical therapy and lifestyle modifications fail. The risks and benefits of such operations were discussed in depth.

During subsequent follow up appointments her symptoms were assessed. The outcome was good and she is currently still undergoing conservative management with regular outpatient clinic follow up.

\section{DISCUSSION}

A good knowledge on the embryology of the aorta and its vessels is pivotal in order to understand the pathogenesis of dysphagia lusoria. The structure of the aortic arch and its major vessels can be abnormal as a consequence of developmental anomalies. These are relatively common; being up to $3 \%$ of people based on autopsy studies. ${ }^{3}$ Bayford first coined the term "dysphagia lusoria" referring to a birth defect encompassing any aortic root anomaly that causes dysphagia. ${ }^{1}$

In normal embryological development there is a duplication system of the aortic arches in which there is a right and left aortic arches. The part proximal to the origins of the right subclavian and 
common carotid arteries undergoes atresia and these two major arteries will then fuse to form what is known as the right brachiocephalic artery. This artery, otherwise also known as the innominate artery, will then be the first branch of the left aortic arch. Therefore at birth, the first 3 branches of the aorta in a normal infant would be the right brachiocephalic artery, the left common carotid artery and the left subclavian artery. ${ }^{2,4}$ In the case of the aberrant right subclavian artery (ARSA), the fourth vascular arch and the right dorsal aorta undergoes involution. In turn, the seventh intersegmental artery persists in its attachment to the descending aorta.

In $80 \%$ of cases it assumes a retrooesophageal position, anterior to the vertebral column, as it exits out of the thoracic cavity into the right upper limb. Rarely, it traverses between the esophagus and the trachea, and in a minority of cases it lies anterior to both the trachea and esophagus. Our patient has an ARSA that is between the oesophagus and the vertebral column thus causing compression that became clinically significant. In a third of cases, the ARSA is associated with carotid artery anomalies with a common origin of the left and right carotid artery also known as bicarotid truncus. ${ }^{3}$

The ARSA, sometimes also known as the "lusorian artery", has a prevalence of $0.4-0.7 \%$ of the population. ${ }^{5,6}$ Despite having a low prevalence, ARSA is reported as being the most common developmental anomaly involving the arch of the aorta. Janssen et al concluded, based on autopsy and retrospective studies that about $60-70 \%$ of patients remain asymptomatic throughout their lives. ${ }^{7}$ In children, the most common presenting history would be of respiratory problems. It is postulated that in children, the trachea is soft and flaccid contributing to airway compression with associated lower tract infections.

In adulthood, patients become symptomatic in the later decades of life. The reason behind this is unclear but it has been proposed that there is increasing rigidity of the oesophagus combined with arterial thickening due to artherosclerosis as age progresses. ${ }^{7}$ As observed in our patient, the dysphagia worsened later in life. Symptomatic patients usually present with dysphagia, stridor, cough, and thoracic chest pain as a results of compression to nearby structures.

Endoscopy findings for ARSA are usually normal, but sometimes a pulsatile impression may be seen. ${ }^{2}$ Barium-contrast examination of the esophagus, showing a characteristic diagonal compression defect at the level of the 3rd and 4th vertebrae, is an excellent tool for detecting this condition. ${ }^{7}$ Computed tomographic scanning, angiography, or both, usually confirm the diagnosis. New imaging techniques such as magnetic resonance imaging may contribute to better visualization, especially when an aneurysm is present in the proximal part of the artery. $^{10}$

Treatment options for ARSA mainly depend on the severity of symptoms and presence of aneurysm. Janssen and colleagues reported three out of six patients with dysphagia lusoria who became free of symptoms after dietary changes or acid inhibition or promotility agents, which is similar with our patient's condition. ${ }^{7}$ The dietary and lifestyle modifications include reducing or avoidance of exacerbating foods, eating slower, adequate chewing, taking smaller bites and more liquids ${ }^{2}$. For patients who remain symptomatic despite conservative measures, surgical intervention is advocated.

The main goal of the operative repair is to remove the aberrant vessel causing the symptoms and restoring the circulation to the right upper limb. The surgical approach will depend on the vascular abnormality. In a patient with a normal aortic arch without aneurysmal formation of the proximal aberrant right subclavian artery, a supraclavicular approach alone is suitable. ${ }^{3}$ A combined cervical and thoracic approach, via a median sternotomy or lateral thoracotomy, would be suitable for patients with other associated lesions ${ }^{8}$.

Kieffer et al reported a case series involving 33 patients who were treated surgically according to the underlying pathology, with proximal occlusion of the lusorian artery either by a cervical approach, median sternotomy, or left- or right-sided thoracotomy, according to the side of the aortic arch. ${ }^{8}$ The perioperative mortality rate in the group of patients with aneurysmal disease of the lusorian artery or an aneurysm at the aortic origin of the lusorian artery was $23.5 \%$.

Endovascular treatment of peripheral arteries or aneurysms of the thoracic or abdominal aorta has become increasingly popular. Kopp et al reported a combined approach of endovascular occlusion of the aortic origin of the lusorian artery with subclavian artery transposition and distal prevertebral occlusion. ${ }^{9}$ This method seems to be a safe and effective alternative for elective treatment, with no perioperative deaths being reported. As this is still a new method, long term data on the efficacy of this approach is lacking.

Our patient has yet to undergo any surgical intervention. She is regularly followed up and the symptoms are currently under control. Any deterioration in symptoms will obviate an updated CT angiography to investigate for possible aneurysm.

\section{CONCLUSION}

Aberrant Right Subclavian Artery is a rare entity and needs to be considered in patients with unexplained chronic dysphagia with normal upper gastrointestinal 
endoscopy. Barium swallow with complementary CT angiogram is the investigative tool of choice. Treatment option depends on the severity of the symptoms. Patients with mild symptoms can be treated conservatively and those with recalcitrant symptoms should be considered for surgery.

\section{REFERENCES}

1. Bayford D. His syndrome and sign of dysphagia lusoria. Annals of the Royal College of Surgeons of England 1979; 61: 63-67.

2. Levitt B, Richter JE. Dysphagia lusoria: a comprehensive review. Diseases of the Esophagus 2007; 20: 455-460.

3. van der Velden AS, B.P., Krasznai AG, van Duijvendijk P, van der Vliet JA, Aberrant insertion of right subclavian artery: an Unusual cause of dysphagia in an adult. J Gastrointest Surg 2009; 13: 2064-2067.

4. Brauner E, L.M., Kremer R, Best LA, Kluger Y, Aberrant right subclavian artery - suggested mechanism for oesophageal foreign body impaction: Case report. World Journal of Emergency Surgery 2011; 6(12): 1-3.

5. Molz G, B.B., Aberrant subclavian artery (arteria lusoria): Sex differences in the prevalence of the various forms of the malformations.

Evaluations of 1378 observations. . Virch Arch A Pathol Anat Histol 1978; 380: 303-315.

6. Fockens P, K.K., Tytgat GNJ, Endosonographic imaging of an aberrant right subclavian (lusorian) artery. Gastrointest Endosc 1996; 43: 419.

7. Janssen M, B.M., Veen HF, Smout AJPM, Bekkers JA, Jonkman JGJ, Ouwendijk RJT, Dysphagia lusoria: Clinical aspects, manometric findings, diagnosis, and therapy. The American Journal of Gastroenterology 2000; 95(6): 1411-1416.

8. Kieffer E, Bahnini A, Koskas F, Aberrant subclavian artery: surgical treatment in thirtythree adult patients. J Vasc Surg 1994; 19: 100109.

9. Kopp R, Wizgall I, Kreuzer E, et al, Surgical and endovascular treatment of symptomatic aberrant right subclavian artery (arteria lusoria). Vascular 2007; 15(2): 84-91.

10. Karcaaltincaba M, Haliloglu M, Ozkan E, Kocak $M$, Akinci D, Ariyurek M. Non-invasive imaging of aberrant right subclavian artery pathologies and aberrant right vertebral artery. Br J Radiol 2009; 82: 73-78. 